\title{
Relevance of arm somatic copy number alterations for oncologic outcomes and tumor immune microenvironment in clear cell renal cell carcinoma
}

\author{
Ying Xiong", Yu Qi", Qi Bai, Yu Xia, Li Liu, Jianming Guo \\ Department of Urology, Zhongshan Hospital, Fudan University, Shanghai 200032, China \\ Contributions: (I) Conception and design: J Guo, L Liu, Y Xiong; (II) Administrative support: J Guo, L Liu; (III) Provision of study materials or \\ patients: Y Xia, Q Bai; (IV) Collection and assembly of data: Y Xiong, L Liu, Y Qi; (V) Data analysis and interpretation: J Guo, Y Xiong, L Liu, Y \\ Qi; (VI) Manuscript writing: All authors; (VII) Final approval of manuscript: All authors. \\ \#These authors contributed equally to this work. \\ Correspondence to: Jianming Guo; Li Liu. Department of Urology, Zhongshan Hospital, Fudan University, Shanghai 200032, China. \\ Email guo.jianming@zs-hospital.sh.cn; liuli901@qq.com.
}

\begin{abstract}
Background: Prognostic value of arm somatic copy number alterations (SCNAs) in clear cell renal cell carcinoma (ccRCC) have not been systematically evaluated in a large cohort. Its association with tumor microenvironment remained unknown.

Methods: We retrospectively correlated arm SCNAs with OS and recurrence free survival (RFS) in a cohort of 524 ccRCC patients. The prognostic landscape of arm SCNA was depicted by bubble heatmap. Associations between arm SCNAs and tumor microenvironment were evaluated by CIBERSORT and Gene Set Enrichment Analysis (GSEA).

Results: We found that amplifications of $1 \mathrm{p}, 3 \mathrm{p}$ and loss of 4p, 4q, 5p, 5q, 11p, 11q, 11q, 13q, 19p were independent adverse risk factor for OS, while amplification of $1 \mathrm{q}$ and deletions of $4 \mathrm{p}, 4 \mathrm{q}, 9 \mathrm{p}, 9 \mathrm{q}$ associated with worse RFS. Loss of $4 \mathrm{q}$ were independent adverse risk factor for OS $(\mathrm{P}=0.012, \mathrm{HR}=1.614)$ and $\mathrm{RFS}$ $(\mathrm{P}=0.001, \mathrm{HR}=2.005)$. It could identify a subset of early stage ccRCC patients with high risk of death and recurrence. CXCL9, CXCL10, CXCL11 mRNA level and CD8+ T cell infiltration were downregulated in ccRCC with $4 \mathrm{q}$ deletion. Patients with high arm SCNA level had shorter OS $(\mathrm{P}=0.005)$ and RFS $(\mathrm{P}=0.001)$. Markers, immune cells and pathways referring to immune suppression were elevated in tumors with high arm SCNA level.

Conclusions: In conclusion, loss of $4 \mathrm{q}$ was an independent adverse risk factor for OS and RFS in ccRCC patients and contributed to cytotoxic cell exclusion via downregulation of CXCL9, CXCL10 and CXCL11. Patients with higher arm SCNAs had worse survival and a more immunosuppressive tumor microenvironment.
\end{abstract}

Keywords: Somatic copy number alterations; prognosis; immune microenvironment; clear cell renal cell carcinoma (ccRCC)

Submitted Jul 09, 2019. Accepted for publication Oct 10, 2019.

doi: $10.21037 /$ atm.2019.10.54

View this article at: http://dx.doi.org/10.21037/atm.2019.10.54

\section{Introduction}

Renal cell carcinoma (RCC) represents $2-3 \%$ adult malignancies and inflicts about 271,000 new patients worldwide every year $(1,2)$. Clear cell renal cell carcinoma
(ccRCC) is the most common histological subtype, accounting for around $70-80 \%$ of all RCC (3). Within this seemingly homogeneous category, tumors exhibited varying survival prospects. Over $50 \%$ of the patients are diagnosed with metastatic disease or develop metastasis 
after curative surgeries (4). Currently there are clear clinical needs at the two extremes of ccRCC risk: those with early stage disease choosing management strategies and those with localized late stage ccRCC who might benefit from adjuvant tyrosine kinase inhibitor (TKI) therapies after curative nephrectomy (5).

It is well established that genetic alterations and a net accumulation are responsible for cancer development and progression (6). Some genetic alterations are significantly associated with stage, grade and prognosis (7). For example, in ccRCC loss of chromosome arms $8 \mathrm{p}, 9 \mathrm{p}$ and $14 \mathrm{q}$ is associated with higher stage and grade; deletions of $9 p$ correlates with early recurrence (8). However, the prognostic value of each arm SCNA in ccRCC has not been systematically evaluated in a large cohort. Besides, it is increasingly recognized that tumor microenvironment plays a fundamental role in tumor progression (9). Previous researches suggested tumor aneuploidy correlated with markers of immune evasion in some malignancies (10). Relations between histologic features, clinical outcomes and cytogenetic aberrations have been studied (11), but further crosstalk between arm SCNA and tumor immune microenvironment in ccRCC remained unknown. Comprehensive genetic profiling and its associations with tumor microenvironment may not only provide insights into the mechanisms of tumor progression, but also provide potential prognostic biomarkers (12).

In this study, we retrospectively correlated arm somatic copy number alterations with clinicopathological information and oncologic outcomes in a cohort of 524 ccRCC patients, demonstrating the prognostic landscape of arm SCNAs. We further analyzed how arm SCNA burden impact patient survival and tumor microenvironment in ccRCC.

\section{Methods}

\section{Patients and data collection}

We retrospectively analyzed the copy number alterations, gene expression profiles and clinical information of 524 ccRCC patients who had undergone nephrectomy in The Cancer Genome Atlas (TCGA) Kidney Clear Cell Renal Cell Carcinoma (KIRC) cohort. Copy number alterations of each sample were downloaded and processed from cBioPortal (http://www.cbioportal.org/). Clinical information of the TCGA KIRC cohort was obtained from the TCGA Pan-cancer Clinical Data Resource, which provides high-quality clinical data (13). We used the UCSC Xena (https://xenabrowser.net/heatmap/) to download RNA-seq data. The mRNA levels were evaluated with bulk tumor samples. Patients with incomplete genetic or clinical information were excluded. Patients who received neoadjuvant therapy were not included neither. The study was approved by the Clinical Research Ethics Committee of Zhongshan Hospital, Fudan University with the approval number B2015-030.

\section{Estimated immune cell type infiltrations}

CIBERSOTR is a computational method for inferring leukocyte representation in bulk tumor transcriptomes and has been proved to have strong agreement with ground truth assessments in bulk tumors $(14,15)$. We used CIBERSORT to calculate the absolute normalized cell count and relative cell fraction of major immune cell types with LM22 gene signature. To support results obtained with CIBERSORT, we also compared the metagene value for Treg cells, macrophages and NK cells with three well-established immune signatures (16). The metagene values were computed to summarize an immune gene signature as the mean value of each gene in the category. Comparison of gene expression profiles of arm SCNA ${ }^{\text {high }}$ versus SCNA $^{\text {low }}$ tumors was carried out with Gene Set Enrichment Analysis (GSEA) to gain more biological understanding of arm SCNAs in ccRCC (17).

\section{Statistical analysis}

Kaplan-Meier analysis with log-rank test demonstrated the survival curves and survival differences between arm $\mathrm{SCNA}^{\text {high }}$ and arm SCNA ${ }^{\text {low }}$ tumors. OS was defined as the time of curative surgery to the time of death or last follow up time, while recurrence free survival (RFS) was calculated as the time from surgery to the time of recurrence or metastasis. Patients with metastatic renal cell carcinoma were excluded from RFS analyses. The prognostic value of arm SCNAs was evaluated by univariate and multivariate analyses. Comparisons of clinicopathological parameters between two groups were carried out by Student $\mathrm{t}$ test or Mann-Whitney $\mathrm{U}$ test depending on normality of the data distribution, Pearson' s chi-square test and Cochran-Mantel-Haenszel $\chi^{2}$ test. All statistical analyses were two-sided and $\mathrm{P}<0.05$ was considered statistically significant. 
A

$$
\begin{aligned}
& \text { - 1p amp } \\
& \text { - 1qamp } \\
& \text { 2p amp } \\
& \text { - } 2 q \text { amp } \\
& \text { - 3p amp } \\
& \text { - } 3 q \text { amp } \\
& \text { - } 4 p \text { amp } \\
& \text { 1. } 4 q a m p \\
& \begin{array}{r}
5 p a m p \\
5 q a m p
\end{array} \\
& \text { 1. } 6 \mathrm{p} a \mathrm{amp} \\
& \text { - } 6 q \text { amp } \\
& \text { 7p amp } \\
& \text { 7qamp } \\
& \text { - } 8 p \text { amp } \\
& \text { - } 8 q a m p \\
& \text { - 9p amp } \\
& \text { - 9q amp } \\
& \text { 110p amp } \\
& \text { 10q amp } \\
& \text { - 11p amp } \\
& \text { - 11q amp } \\
& 12 p \text { amp } \\
& 12 q \mathrm{amp} \\
& \text { - 13q amp } \\
& \text {-14q amp } \\
& \text { - 15q amp } \\
& 16 p \text { amp } \\
& \text { 16q amp } \\
& \text { - 17p amp } \\
& \text { - 17q amp } \\
& \text { 18p amp } \\
& \text { - 18q amp } \\
& 19 p \text { amp } \\
& 19 q \text { amp } \\
& \text { 20p amp } \\
& 20 q \text { amp } \\
& \text { 21q amp } \\
& \text { 22q amp }
\end{aligned}
$$
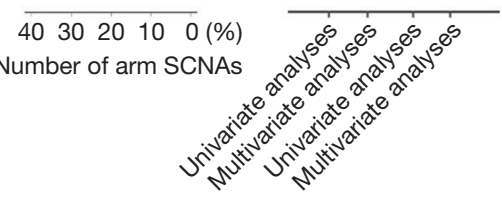

$\overline{\text { OS }} \overline{\text { RFS }}$

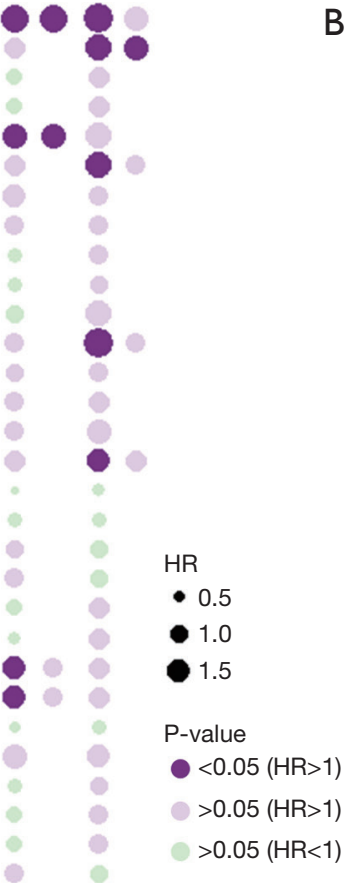

B

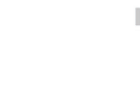

$1 q$ del

$2 p$ del

I $2 q$ del

3p del

$3 q$ del

- $4 p$ del

- $4 q \mathrm{del}$

I $5 p$ del

I $5 q$ del

$6 \mathrm{p} \mathrm{del}$

$6 q$ del

- $8 p$ del

- $8 \mathrm{qde}$

- 9p del

9q de

10p de

$10 q$ del

$11 \mathrm{p}$ del

$111 \mathrm{q}$ del

-13q del

$14 q$ del

$15 q$ del

| 16p del

116q del

17p del

$17 q$ del

18p de

-18q del

119p del

| 20p del

21q del

22q del

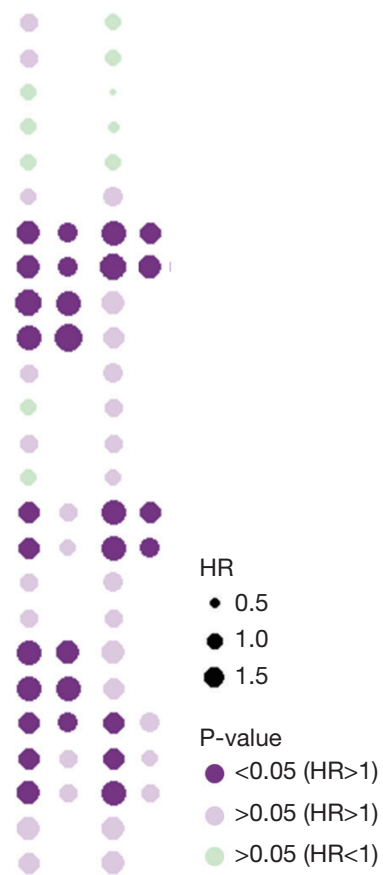

$604020 \quad 0(\%)$ Number of arm SCNAs

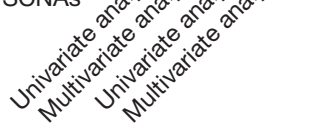

$\overline{\text { OS }} \overline{\text { RFS }}$

Figure 1 Bubble heatmaps demonstrate the prognostic landscape of arm somatic copy number alterations in ccRCC. (A) Prevalence of arm somatic copy number amplifications and their association with OS and RFS; (B) prevalence of arm somatic copy number deletions and their association with OS and RFS. A dark purple bubble indicates that the arm SCNA significantly associates with shorter OS or RFS, while a light purple bubble indicates that the arm SCNA associates with shorter OS or RFS without statistical significance. Likewise, a light green bubble indicates that the arm SCNA associates with prolonged OS or RFS without statistical significance. The size of the bubble indicates the hazard ratio. Amp, amplification; Del, deletion; RFS, recurrence-free survival; SCNA, somatic copy number alteration; ccRCC, clear cell renal cell carcinoma.

\section{Results}

\section{Bubble heatmaps demonstrate the prevalence and prognostic landscape of arm somatic copy number alterations in ccRCC}

Figure 1 shows the percentage of arm SCNAs and their prognostic value with univariate as well as multivariate analysis respectively. Arm SCNAs present in less than 1\% ccRCC samples were not displayed. Amplification of $5 \mathrm{q}$ (39.7\%), 5p (36.1\%), 7p (32.6\%), 7q (32.6\%), 12q (23.1\%), $12 \mathrm{p}(23.1 \%), 20 \mathrm{q}(21.9 \%)$ and $20 \mathrm{p}(21.4 \%)$ occurred in over $20 \%$ of ccRCC (Figure $1 A$ ). In the bubble heatmaps, a 
dark purple bubble indicated a significant association with worse OS or RFS while the size of the bubble indicated the hazard ratio (HR). In univariate analysis of copy number amplification for OS, $1 \mathrm{p}(\mathrm{P}=0.002, \mathrm{HR}=2.497), 3 \mathrm{p}$ $(\mathrm{P}=0.041, \mathrm{HR}=1.947), 12 \mathrm{p}(\mathrm{P}=0.009, \mathrm{HR}=1.555)$ and $12 \mathrm{q}$ $(\mathrm{P}=0.013, \mathrm{HR}=1.520)$ were associated with shorter OS. For RFS, 1p ( $\mathrm{P}=0.011, \mathrm{HR}=2.538)$, 1q $(\mathrm{P}=0.002, \mathrm{HR}=2.163)$, $3 \mathrm{q}(\mathrm{P}=0.002, \mathrm{HR}=2.042), 6 \mathrm{q}(\mathrm{P}=0.030, \mathrm{HR}=2.696), 8 \mathrm{q}$ $(\mathrm{P}=0.036, \mathrm{HR}=1.69), 19 \mathrm{p}(\mathrm{P}=0.010, \mathrm{HR}=1.859), 19 \mathrm{q}$ $(\mathrm{P}=0.003, \mathrm{HR}=1.985)$ and $20 \mathrm{q}(\mathrm{P}=0.028, \mathrm{HR}=1.584)$ were adverse risk factors. The prognostic value of arm SCNA was further assessed by multivariable cox regression analyses each, with stage and grade as covariates. Amplification of $1 \mathrm{p}(\mathrm{P}=0.012, \mathrm{HR}=2.11), 3 \mathrm{p}(\mathrm{P}=0.034, \mathrm{HR}=2.01)$ and $1 \mathrm{q}$ $(\mathrm{P}=0.022, \mathrm{HR}=1834)$ were independent adverse risk factor for OS or RFS in multivariate analysis (Figure $1 A$ ).

The most frequent arm somatic copy number loss is $3 \mathrm{p}$ (71.9\%), followed by $14 q(40.4 \%), 9 q(28.6 \%), 9 p(28.1 \%)$, $8 \mathrm{p}(26.0 \%)$ and $6 \mathrm{q}(24.0 \%)$ (Figure $1 B)$. We found that loss of $4 \mathrm{p}$ (OS: $\mathrm{P}<0.001, \mathrm{HR}=2.024$; RFS: $\mathrm{P}<0.001, \mathrm{HR}=2.57$ ), 4q (OS: $\mathrm{P}<0.001, \mathrm{HR}=2.294$; RFS: $\mathrm{P}<0.001, \mathrm{HR}=3.13$ ), 9p (OS: $\mathrm{P}<0.001, \mathrm{HR}=1.898$; RFS: $\mathrm{P}<0.001, \mathrm{HR}=1.745$ ), 9q (OS: $\mathrm{P}<0.001, \mathrm{HR}=1.745 ; \mathrm{RFS}: \mathrm{P}<0.001, \mathrm{HR}=2.624)$, 13q (OS: $\mathrm{P}=0.001, \mathrm{HR}=1.895$; RFS: $\mathrm{P}=0.018$, HR =1.731), 14q (OS: $\mathrm{P}=0.002, \mathrm{HR}=1.623$; RFS: $\mathrm{P}=0.004$, HR =1.681), 15q (OS: $\mathrm{P}=0.002, \mathrm{HR}=2.158$; RFS: $\mathrm{P}<0.001, \mathrm{HR}=2.695)$, $18 \mathrm{p}(\mathrm{OS}: \mathrm{P}=0.002, \mathrm{HR}=1.766$; RFS: $\mathrm{P}=0.049, \mathrm{HR}=1.548)$ and $22 \mathrm{q}(\mathrm{OS}: \mathrm{P}=0.008, \mathrm{HR}=1.851$; RFS: $\mathrm{P}=0.003$, HR $=2.240$ ) associated with worse OS and RFS in univariate analyses. After adjustment for stage and grade, deletion of $4 \mathrm{p}(\mathrm{P}=0.018, \mathrm{HR}=1.558), 4 \mathrm{q}(\mathrm{P}=0.012, \mathrm{HR}=1.614)$ and $13 \mathrm{q}$ $(\mathrm{P}=0.021, \mathrm{HR}=1.588)$ remained their statistical significance for OS while 4p $(\mathrm{P}=0.005, \mathrm{HR}=1.858), 4 \mathrm{q}(\mathrm{P}=0.001, \mathrm{HR}$ $=2.005), 9 \mathrm{p}(\mathrm{P}=0.013, \mathrm{HR}=1.629)$ and $9 \mathrm{q}(\mathrm{P}=0.020$, HR $=1.583$ ) remained significant as an independent adverse risk factor for RFS (Figure 1B).

\section{Loss of $4 q$ contributes to $C D 8+T$ cell exclusion and identifies a subset of early stage patients with bigh risk of death and recurrence}

The findings suggested that deletion of $4 q$ and $4 p$ are the only independent adverse risk factors for both OS and RFS. Genes encoding chemoattractant for major cytotoxic CD8+T cells and NK cells, CXCL9, CXCL10 and CXCL11 all located on 4q.21. We found that CXCL9 $(P=0.029)$, CXCL10 ( $\mathrm{P}=0.059)$ and CXCL11 $(\mathrm{P}=0.017) \mathrm{mRNA}$ expression were all significantly downregulated in ccRCC tumors with $4 \mathrm{q}$ deletion (Figure $2 A, B, C$ ). CIBERSORT analysis revealed that $\mathrm{CD} 8+\mathrm{T}$ cell infiltration $(\mathrm{P}=0.002)$ and NK cell infiltration $(\mathrm{P}=0.051)$ decreased in ccRCC tumors with loss of $4 \mathrm{q}$ (Figure 2D,E). These findings suggested that $4 \mathrm{q}$ deletion led to immune exclusion via downregulation of CXCL9, CXCL10 and CXCL11.

There are clear clinical needs for identifying early stage patients with high risk of metastasis and late stage patients who might benefit from adjuvant TKI treatment, so we further explored the prognostic value of $4 q$ and $4 p$ in different stages of ccRCC patients. Analysis of KaplanMeier $\log$-rank test revealed that deletion of $4 \mathrm{q}$ associated with both worse OS $(\mathrm{P}=0.004)$ and RFS $(\mathrm{P}=0.007)$ in early stage (I \& II) ccRCC patients (Figure 2F,G). Among early stage ccRCC patients, multivariate analyses showed that $4 \mathrm{q}$ deletion remained an independent risk factor for OS $(\mathrm{P}=0.018, \mathrm{HR}=2.572,95 \% \mathrm{CI}: 1.173-5.639)$ and $\mathrm{RFS}$ $(\mathrm{P}=0.043, \mathrm{HR}=2.634$, 95\% CI: 1.033-6.721) when factoring in tumor grade and stage. Thus, loss of $4 \mathrm{q}$ was a promising biomarker that could identify a subset of early stage ccRCC patients with a high risk of death and recurrence. Besides, in stage III \& IV patients, 4q loss indicated a shorter RFS (Figure $2 H, I)$. Stage I \& II patients with $4 \mathrm{p}$ deletion had shorter OS (Figure $S 1 A, B$ ) while stage III \& IV with 4p deletion had worse RFS (Figure $S 1 C, D)$.

\section{High level of arm copy number alterations associates with worse survival and higher tumor mutation burden}

Number of arm SCNAs was split at median. Tumor with six or more arm SCNAs was considered arm SCNA ${ }^{\text {high }}$ while tumors with less than six arm SCNAs was considered arm $\mathrm{SCNA}^{\text {low }}$. We analyzed the correlations between clinicopathological characteristics and arm SCNA level. Tumors of people over 65 years old $(\mathrm{P}=0.028)$ and males $(\mathrm{P}=0.036)$ tended to harbor high arm SCNA level. High arm SCNA level also associated with higher tumor stage $(\mathrm{P}<0.001)$ and grade $(\mathrm{P}<0.001)$ (Table S1). Late stage and high grade ccRCC had more copy number alterations compared with early stage $(\mathrm{P}<0.001)$ and low grade $(\mathrm{P}<0.001)$ tumors, respectively (Figure $3 A, B)$. In ccRCC, tumor mutation burdens are more abundant in arm SCNA $^{\text {high }}$ tumors $(\mathrm{P}=0.028)$, in contrast to a previous study showing that there are more SCNAs in tumors with low mutation burden (18) (Figure 3C). Kaplan-Meier analysis showed that arm $\mathrm{SCNA}^{\text {low }}$ patients had significantly prolonged OS $(\mathrm{P}=0.005)$ and $\mathrm{RFS}(\mathrm{P}=0.001)$ compared with arm SCNA ${ }^{\text {high }}$ patients (Figure $3 D, E$ ). 

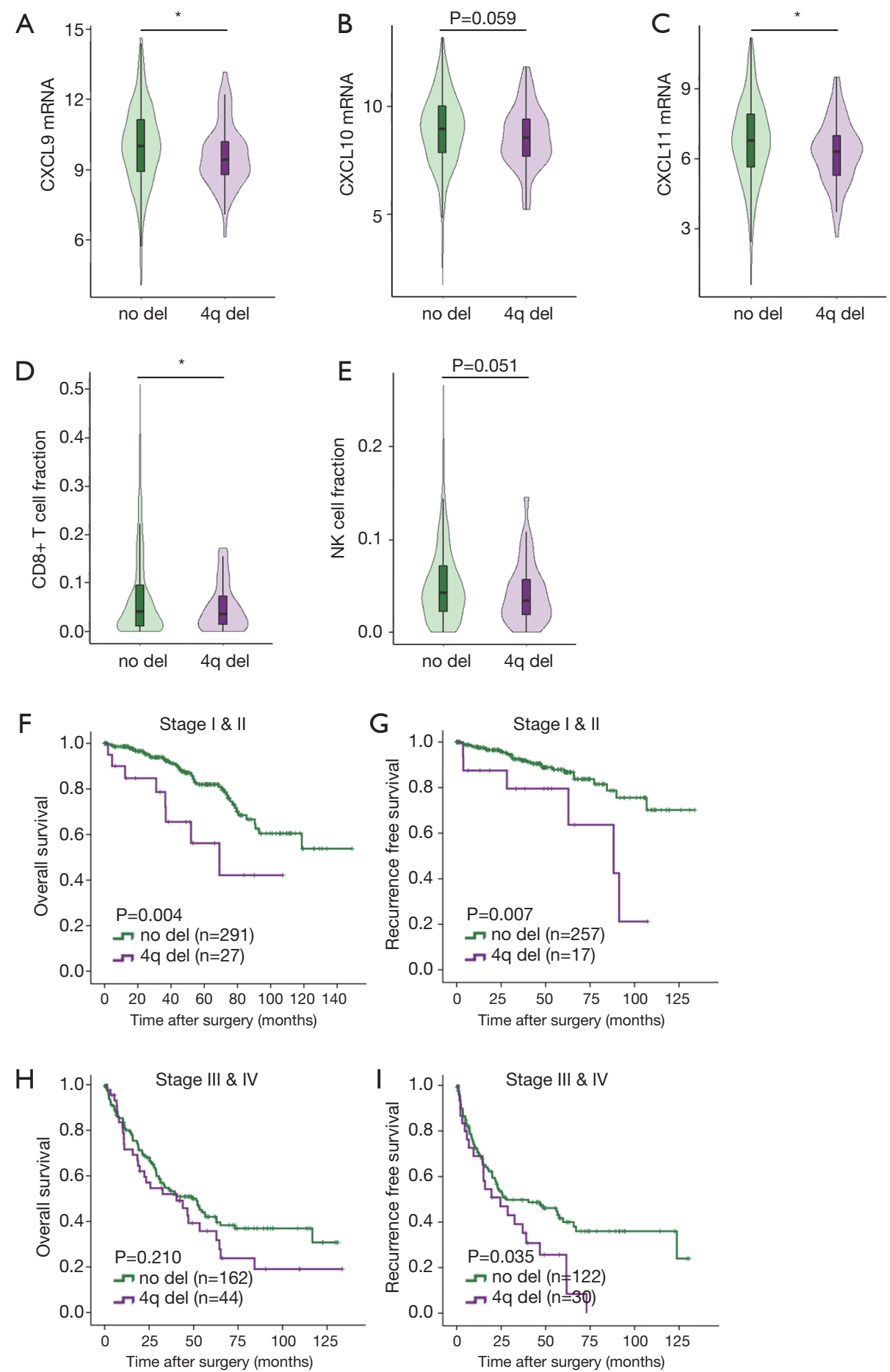

Figure 2 Loss of 4q contributes to CD8+ T cell exclusion and identifies a subset of early stage patients with high risk of death and recurrence. (A) CXCL9 mRNA expression in patients with or without 4q deletion; (B) CXCL10 mRNA expression in patients with or without $4 \mathrm{q}$ deletion; (C) CXCL11 mRNA expression in patients with or without 4q deletion; (D) CD8+ T cell infiltration in patients with or without $4 \mathrm{q}$ deletion; (E) NK cell infiltration in patients with or without $4 \mathrm{q}$ deletion; (F) Kaplan-Meier analysis of $4 \mathrm{q}$ deletion for OS in stage I \& II patients; (G) Kaplan-Meier analysis of 4q deletion for RFS in stage I \& II patients; (H) Kaplan-Meier analysis of 4q deletion for OS in stage III \& IV patients; (I) Kaplan-Meier analysis of 4q deletion for RFS in stage III \& IV patients. * $\mathrm{P}<0.05$. Del, deletion; RFS, recurrence-free survival. 

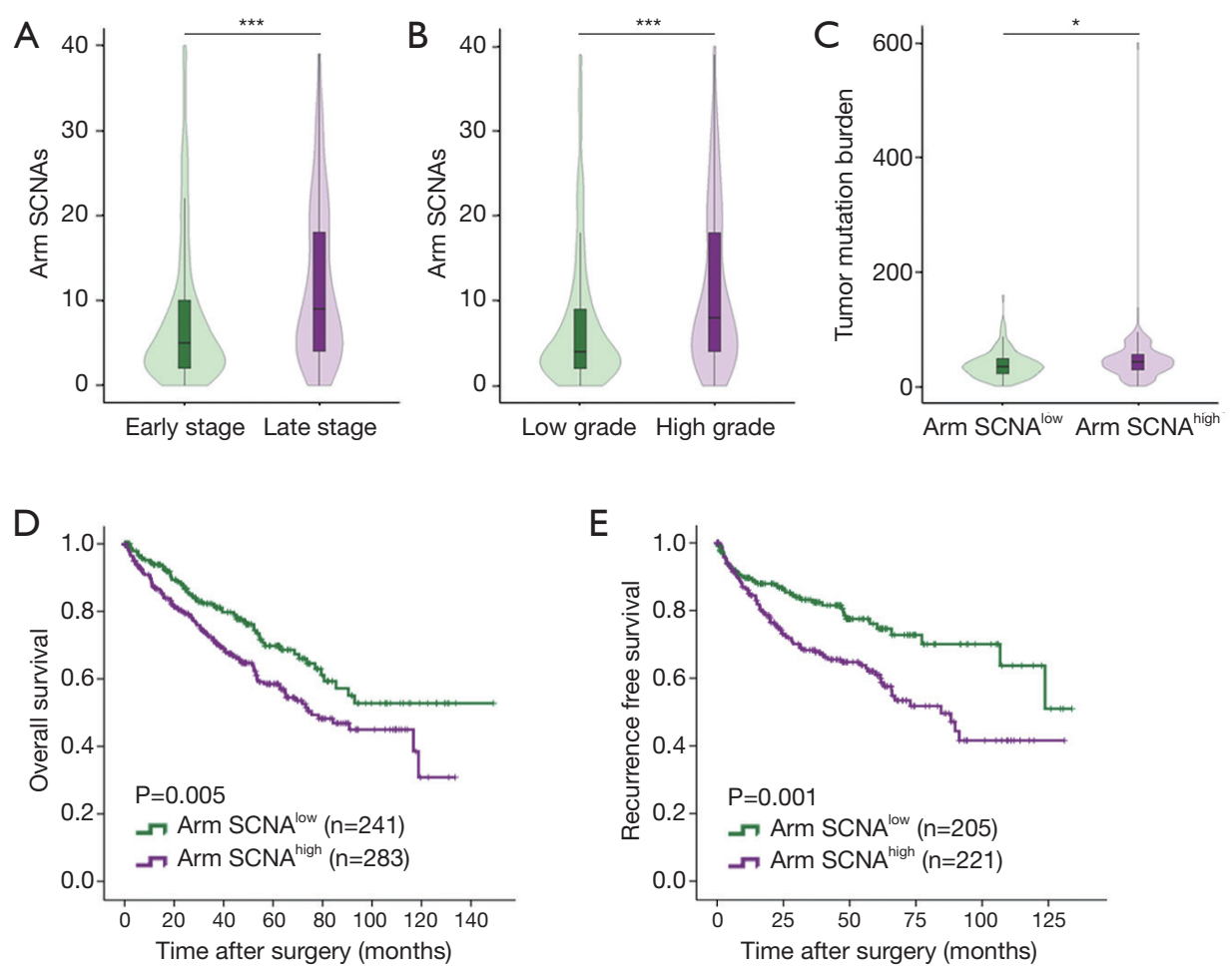

Figure 3 High level of arm copy number alterations associates with worse survival and higher tumor mutation burden. (A) Number of arm SCNAs in early stage (I \& II) and late stage (III \& IV) ccRCC patients; (B) number of arm SCNAs in low grade (I \& II) and high grade (III \& IV) ccRCC patients; (C) tumor mutation burdens in arm SCNA ${ }^{\text {high }}$ and SCNA ${ }^{\text {low }}$ tumors; (D) Kaplan-Meier analysis of arm SCNA level for OS in ccRCC patients; (E) Kaplan-Meier analysis of arm SCNA level for RFS in ccRCC patients. *, $\mathrm{P}<0.05$; ***, P<0.001. SCNA, somatic copy number alteration; ccRCC, clear cell renal cell carcinoma.

\section{Immunosuppressive immune cells and T cell exhaustion markers enriched in high arm copy number alteration patients}

ccRCCs are highly immunogenic tumors. We wondered whether arm SCNA could imparted different features on the tumor microenvironment. CIBERSORT analysis was performed to unveil the immune cell composition in arm SCNA $^{\text {high }}$ and arm SCNA ${ }^{\text {low }}$ tumors. Treg cells, macrophages and TFH cells significantly enriched in arm SCNA ${ }^{\text {high }}$ tumors in terms of both absolute cell count and relative cell fraction. On the other hand, NK cells and mast cells were reduced in arm SCNA ${ }^{\text {low }}$ tumors (Figure $4 A$ ). To confirm our findings, we evaluated the established metagene value for Treg cells, macrophages and NK cells, which had been well recognized for their pro- or anti-tumor abilities. In accordance with CIBERSORT analysis, gene signature of Treg cells and macrophages were significantly elevated in arm SCNA ${ }^{\text {high }}$ tumors (Figure 4B,C). NK cells metagene values were downregulated in arm SCNA ${ }^{\text {high }}$ tumors (Figure $4 D$ ).
In most malignancies, higher CD8+ T cell infiltration associated with prolonged survival. However, in ccRCC, higher CD8+ T cell infiltration was an adverse risk factor for patient survival (19). We did not observe any changes in CD $8+T$ cell infiltration. Instead, markers of T cell exhaustion including PD-1, CTLA-4, LAG-3 and TIGIT significantly elevated in arm SCNA ${ }^{\text {high }}$ tumors (Figure 4E, $F, G, H$ ), which indicated that the antitumor immunity of CD8+ T cells in arm SCNA ${ }^{\text {high }}$ tumors were largely offset by immunosuppressive elements in tumor microenvironment. GSEA analyses revealed that lymphocyte mediated immunity was down-regulated in arm SCNA ${ }^{\text {high }}$ tumors (Figure 4I). Our findings suggested that arm SCNAs were closely associated with immune suppression in ccRCC.

\section{Discussion}

To our knowledge, this is the first study to systematically 
A
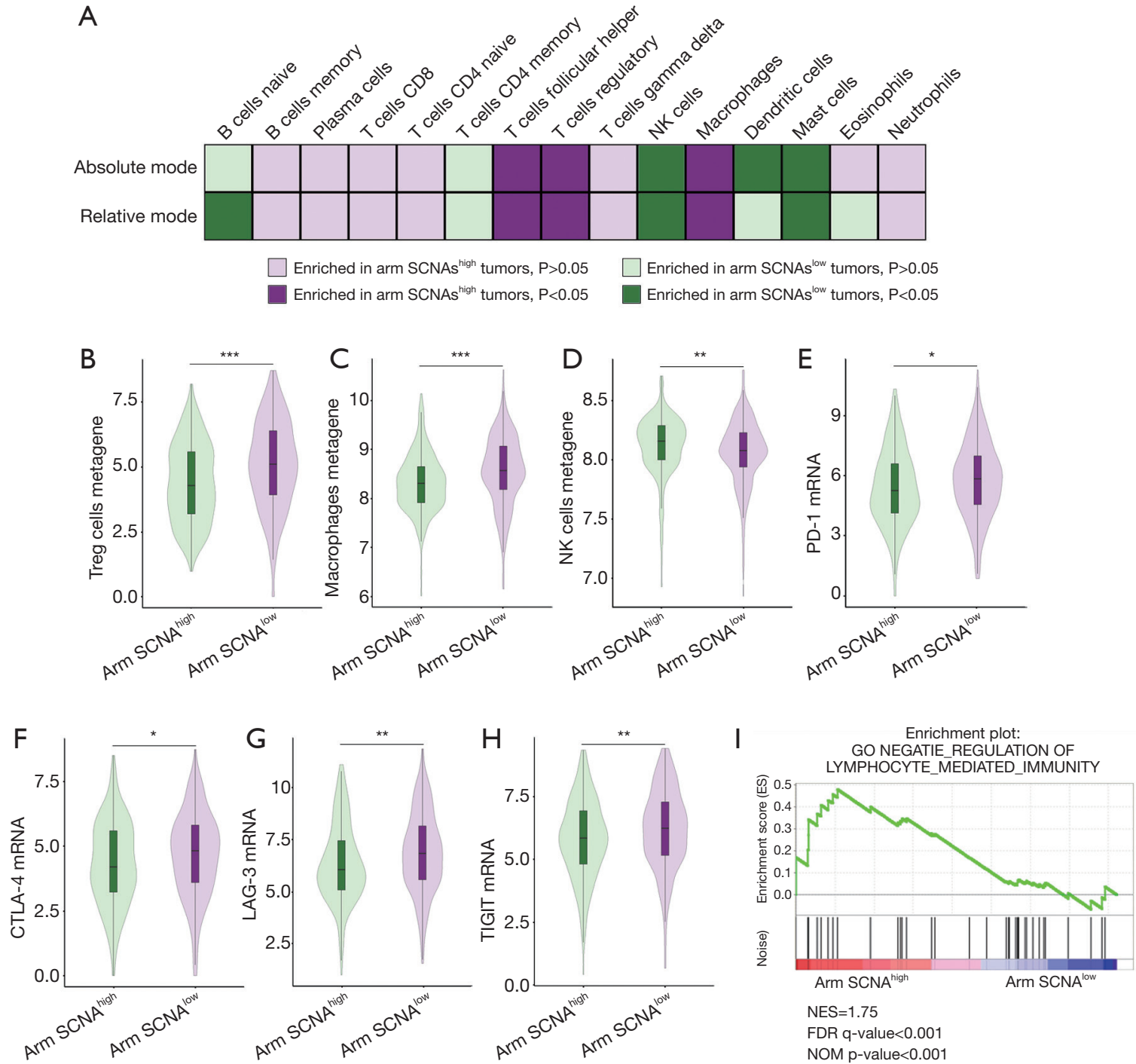

Figure 4 Immunosuppressive immune cells and $\mathrm{T}$ cell exhaustion markers enriched in patients with high level of arm copy number alterations. (A) Different features of immune cell composition in arm SCNA ${ }^{\text {high }}$ and arm SCNA ${ }^{\text {low }}$ tumors; (B) Treg cells metagene values in arm SCNA ${ }^{\text {high }}$ and arm SCNA ${ }^{\text {low }}$ tumors; (C) Macrophages metagene values in arm SCNA ${ }^{\text {high }}$ and arm SCNA ${ }^{\text {low }}$ tumors; (D) NK cells metagene values in arm SCNA ${ }^{\text {high }}$ and arm SCNA ${ }^{\text {low }}$ tumors; (E) PD-1 mRNA level in arm SCNA ${ }^{\text {high }}$ and arm SCNA ${ }^{\text {low }}$ tumors; (F) CTLA-4 mRNA level in arm SCNA ${ }^{\text {high }}$ and arm SCNA ${ }^{\text {low }}$ tumors; (G) LAG-3 mRNA level in arm SCNA ${ }^{\text {high }}$ and arm SCNA ${ }^{\text {low }}$ tumors; (H) TIGIT mRNA level in arm SCNA ${ }^{\text {high }}$ and arm SCNA ${ }^{\text {low }}$ tumors; (I) gene set enrichment analysis of "NEGATIE_REGULATION OF LYMPHOCYTE_MEDIATED_IMMUNITY" pathway in arm SCNA ${ }^{\text {high }}$ versus arm SCNA ${ }^{\text {low }}$ tumors. **, $\mathrm{P}<0.01$. SCNA, somatic copy number alteration. *, $\mathrm{P}<0.05 ;{ }^{* *}, \mathrm{P}<0.01 ;{ }^{* * *}, \mathrm{P}<0.001$.

evaluate the prognostic landscape of arm SCNAs and the associations with tumor microenvironment in a large cohort of over $500 \mathrm{ccRCC}$ patients. The most frequent alterations included deletions of $3 p, 14 q$ and gain of $5 q$, in complete accordance with previous studies (20). The von Hippel Lindau (VHL) gene is one of the most important tumor suppressor gene located at $3 \mathrm{p} 25.3$ region in ccRCC. There is also a high concordance of other chromosome aberrations such as loss of 6q, 8p, 9q and amplification of 7p, 7q, 12q. In ccRCC, some arm SCNAs were associated with clinicopathological features. Significant associations with late tumor stage or grade were observed for loss of 9p, 9q, 14q, 18p, 21q (12). There was a 
high rate of chromosomal imbalances with loss of 9q, 15q, 18p, 18q, 22q and gains of 1q, 8q in RCC with sarcomatoid differentiations (21). In this study, we found almost all of these arm SCNAs correlated with worse OS or RFS in univariate analyses except loss of 21q.

According to previous studies that included survival as an end point, deletions of $4 p, 8 p, 9 p, 14 q$ and gain of $8 q$ have been suggested to correlate with poorer survival (11,22-24). Loss of $4 p, 9 p$ maintained as independent adverse risk factors when factoring stage and grade with multivariate analyses in our study. Furthermore, we identified that $4 \mathrm{q}$ as an independent adverse risk factor for both OS and RFS in ccRCC. It could also stratify a subset of early stage ccRCC patients with a higher risk of death and recurrence. The prognostic value of $4 \mathrm{q}$ deletion has not been discovered before, partly because of the limited sample size and inappropriate statistical power in previous studies. Chemokines responsible for CD8+ T cell recruitment including CXCL9, CXCL10 and CXCL11 located on chromosome 4q. Expression analysis reveal that CXCL9, CXCL10 and CXCL11 were downregulated in ccRCC with $4 p$ deletions. CD8+ T cell infiltration significantly deceased in $4 \mathrm{q}$ deletion tumors. These findings indicated that loss of $4 \mathrm{q}$ contributed to $\mathrm{CD} 8+\mathrm{T}$ cell exclusion via downregulation of CXCL9, CXCL10 and CXCL11, which led to worse survival in patients with $4 \mathrm{q}$ deletions. In contrary to previous reports that gain of $5 q$ and deletion of $3 p$ were associated with better prognosis, we found that no arm SCNA correlated with prolonged survival with statistical significance $(11,25)$.

Tumors with high arm SCNA level harbored higher tumor mutation burden, elevated infiltrations of macrophages and Treg cells. Treg cells and tumor associated macrophages contributed substantially to the suppression of antitumor $\mathrm{T}$ cell responses as they frequently accumulate in the tumor microenvironment (26). On the contrary, there were fewer NK cells and mast cells in arm SCNA ${ }^{\text {high }}$ tumors. NK cells are considered important anti-cancer effector cells, as they can kill neoplastic cells presenting the MHC class I molecule (9). Mast cells could boost anti-tumor immunity and associate with prolonged survival in ccRCC as well (27). Markers and pathways referring to immune evasion were also upregulated in tumors with more arm SCNAs. Arm SCNA level correlated with markers of immune evasion and immunosuppressive immune cell infiltration, consistent with a mechanism related to gene dosage imbalance instead of functions of certain genes. Our findings suggested that arm SCNAs were closely associated with immune suppression in ccRCC. The altered tumor microenvironment conferred by
$4 \mathrm{q}$ loss might also suggested a potential link between $4 \mathrm{q}$ loss and immunotherapy treatment response for metastatic renal cell carcinoma. Genomic instability and immune evasion in arm $\mathrm{SCNA}^{\text {high }}$ tumors may contribute to the shortened survival in patients with high arm SCNA level.

However, some major limitations remained. This is a retrospective study without external validation. Cohort specific biases including patient ethnicity/race and clinical practice at the institution could affect our conclusions. These results will need to be further validated and confirmed in prospective randomized clinical trials.

\section{Conclusions}

In conclusion, we demonstrated the prognostic landscape of each arm SCNA in ccRCC and identified $4 \mathrm{q}$ deletion as independent adverse risk factors for both OS and RFS. Early stage ccRCC patients with 4q deletion had higher risk of death and recurrence. Loss of $4 \mathrm{q}$ contributed to cytotoxic cells exclusion via down regulation of CXCL9, CXCL10 and CXCL11. Higher arm SCNA burden correlated with worse survival and a more immunosuppressive tumor microenvironment.

\section{Acknowledgments}

Funding: This study was funded by grants from National Natural Science Foundation of China (81702496, 81702497, 81702805 and 81772696). All these study sponsors have no roles in the study design, in the collection, analysis, and in the interpretation of data.

\section{Footnote}

Conflicts of Interest: The authors have no conflicts of interest to declare.

Ethical Statement: The authors are accountable for all aspects of the work in ensuring that questions related to the accuracy or integrity of any part of the work are appropriately investigated and resolved. The study was approved by the Clinical Research Ethics Committee of Zhongshan Hospital, Fudan University with the approval number B2015-030.

\section{References}

1. Ferlay J, Shin HR, Bray F, et al. Estimates of worldwide 
burden of cancer in 2008: GLOBOCAN 2008. Int J

Cancer 2010;127:2893-917.

2. Siegel RL, Miller KD, Jemal A. Cancer statistics, 2019. CA Cancer J Clin 2019;69:7-34.

3. Cohen HT, McGovern FJ. Renal-cell carcinoma. N Engl J Med 2005;353:2477-90.

4. Meskawi M, Sun M, Trinh QD, et al. A review of integrated staging systems for renal cell carcinoma. Eur Urol 2012;62:303-14.

5. Motzer RJ, Ravaud A, Patard JJ, et al. Adjuvant Sunitinib for High-risk Renal Cell Carcinoma After Nephrectomy: Subgroup Analyses and Updated Overall Survival Results. Eur Urol 2018;73:62-8.

6. Cahill DP, Kinzler KW, Vogelstein B, et al. Genetic instability and darwinian selection in tumours. Trends Cell Biol 1999;9:M57-60.

7. Friedlander ML, Hedley DW, Taylor IW. Clinical and biological significance of aneuploidy in human tumours. J Clin Pathol 1984;37:961-74.

8. Moch H, Presti JC, Jr., Sauter G, et al. Genetic aberrations detected by comparative genomic hybridization are associated with clinical outcome in renal cell carcinoma. Cancer Res 1996;56:27-30.

9. Palucka AK, Coussens LM. The Basis of Oncoimmunology. Cell 2016;164:1233-47.

10. Davoli T, Uno H, Wooten EC, et al. Tumor aneuploidy correlates with markers of immune evasion and with reduced response to immunotherapy. Science 2017. doi: 10.1126/science.aaf8399.

11. Klatte T, Rao PN, de Martino M, et al. Cytogenetic profile predicts prognosis of patients with clear cell renal cell carcinoma. J Clin Oncol 2009;27:746-53.

12. Chen M, Ye Y, Yang H, et al. Genome-wide profiling of chromosomal alterations in renal cell carcinoma using high-density single nucleotide polymorphism arrays. Int J Cancer 2009; 125:2342-8.

13. Liu J, Lichtenberg T, Hoadley KA, et al. An Integrated TCGA Pan-Cancer Clinical Data Resource to Drive High-Quality Survival Outcome Analytics. Cell 2018;173:400-16.e11.

14. Gentles AJ, Newman AM, Liu CL, et al. The prognostic landscape of genes and infiltrating immune cells across human cancers. Nat Med 2015;21:938-45.

15. Newman AM, Liu CL, Green MR, et al. Robust enumeration of cell subsets from tissue expression profiles. Nat Methods 2015;12:453-7.

16. Bindea G, Mlecnik B, Tosolini M, et al. Spatiotemporal dynamics of intratumoral immune cells reveal the immune landscape in human cancer. Immunity 2013;39:782-95.

17. Subramanian A, Tamayo P, Mootha VK, et al. Gene set enrichment analysis: a knowledge-based approach for interpreting genome-wide expression profiles. Proc Natl Acad Sci U S A 2005;102:15545-50.

18. Ciriello G, Miller ML, Aksoy BA, et al. Emerging landscape of oncogenic signatures across human cancers. Nat Genet 2013;45:1127-33.

19. Fridman WH, Zitvogel L, Sautes-Fridman C, et al. The immune contexture in cancer prognosis and treatment. Nat Rev Clin Oncol 2017;14:717-34.

20. Moore LE, Jaeger E, Nickerson ML, et al. Genomic copy number alterations in clear cell renal carcinoma: associations with case characteristics and mechanisms of VHL gene inactivation. Oncogenesis 2012;1:e14.

21. Ito T, Pei J, Dulaimi E, et al. Genomic Copy Number Alterations in Renal Cell Carcinoma with Sarcomatoid Features. J Urol 2016;195:852-8.

22. Klatte T, Kroeger N, Rampersaud EN, et al. Gain of chromosome $8 \mathrm{q}$ is associated with metastases and poor survival of patients with clear cell renal cell carcinoma. Cancer 2012;118:5777-82.

23. Elfving P, Mandahl N, Lundgren R, et al. Prognostic implications of cytogenetic findings in kidney cancer. Br J Urol 1997;80:698-706.

24. La Rochelle J, Klatte T, Dastane A, et al. Chromosome $9 p$ deletions identify an aggressive phenotype of clear cell renal cell carcinoma. Cancer 2010;116:4696-702.

25. Gunawan B, Huber W, Holtrup M, et al. Prognostic impacts of cytogenetic findings in clear cell renal cell carcinoma: gain of 5q31-qter predicts a distinct clinical phenotype with favorable prognosis. Cancer Res 2001;61:7731-8.

26. Speiser DE, Ho PC, Verdeil G. Regulatory circuits of T cell function in cancer. Nat Rev Immunol 2016;16:599-611.

27. Fu H, Zhu Y, Wang Y, et al. Tumor Infiltrating Mast Cells (TIMs) Confers a Marked Survival Advantage in Nonmetastatic ClearCell Renal Cell Carcinoma. Ann Surg Oncol 2017;24:1435-42.

Cite this article as: Xiong Y, Qi Y, Bai Q, Xia Y, Liu L, Guo J. Relevance of arm somatic copy number alterations for oncologic outcomes and tumor immune microenvironment in clear cell renal cell carcinoma. Ann Transl Med 2019;7(22):646. doi: 10.21037/atm.2019.10.54 

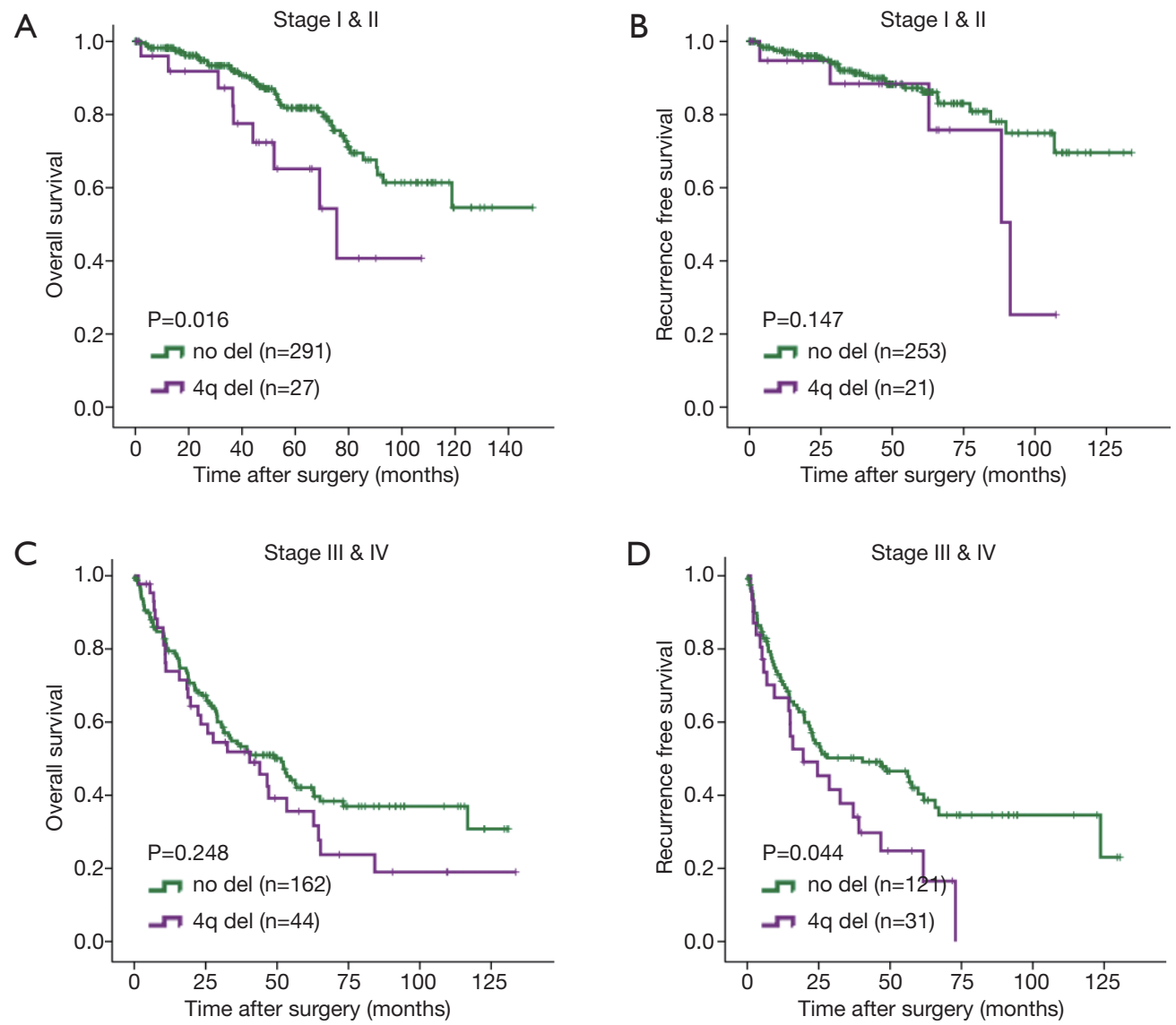

Figure S1 Prognostic value of $4 p$ deletion in ccRCC patients with different stages. (A) Kaplan-Meier analysis of 4p deletion for OS in stage I \& II patients; (B) Kaplan-Meier analysis of 4p deletion for RFS in stage I \& II patients; (C) Kaplan-Meier analysis of 4p deletion for OS in stage III \& IV patients; (D) Kaplan-Meier analysis of 4p deletion for RFS in stage III \& IV patients.

Table S1 Clinical characteristics of ccRCC patients according arm SCNAs level

\begin{tabular}{|c|c|c|c|c|c|}
\hline \multirow{2}{*}{ Characteristics } & \multicolumn{2}{|c|}{ Patients } & \multicolumn{3}{|c|}{ Arm SCNAs level } \\
\hline & $\mathrm{n}$ & $\%$ & Low & High & $P$ value \\
\hline All patients & 524 & 100 & 241 & 283 & \\
\hline Age, years & & & & & $0.028^{\star}$ \\
\hline$<65$ & 328 & 62.6 & 163 & 165 & \\
\hline$\geq 65$ & 196 & 37.4 & 78 & 118 & \\
\hline Gender & & & & & $0.036^{*}$ \\
\hline Female & 186 & 35.5 & 97 & 89 & \\
\hline Male & 338 & 64.5 & 144 & 194 & \\
\hline TNM stage & & & & & $<0.001^{\dagger}$ \\
\hline 1 & 261 & 49.8 & 146 & 115 & \\
\hline ॥ & 57 & 10.9 & 29 & 28 & \\
\hline III & 124 & 23.7 & 43 & 81 & \\
\hline IV & 82 & 15.6 & 23 & 59 & \\
\hline Tumor nucleus grade & & & & & $<0.001^{\dagger}$ \\
\hline 1 & 14 & 2.7 & 13 & 1 & \\
\hline ॥ & 225 & 43.6 & 126 & 99 & \\
\hline III & 202 & 39.1 & 82 & 120 & \\
\hline IV & 75 & 14.5 & 17 & 58 & \\
\hline
\end{tabular}

${ }^{*}, \chi^{2}$ test; ${ }^{\dagger}$, Cochran-Mantel-Haenszel $\chi^{2}$ test. ccRCC, clear cell renal cell carcinoma; SCNAs, somatic copy number alterations. 